\title{
Clustering collaborative filtering approach for Diftari E-Learning platform' recommendation system.
}

\author{
Jamal Mawanel \\ Department of computer science \\ Faculty of Sciences and Technics of \\ Mohammedia, Hassan II University \\ Morocco \\ ja.mawane@gmail.com
}

\author{
Abdelwahab Naji \\ Department of computer science \\ Faculty of Sciences and Technics of \\ Mohammedia, Hassan II University \\ Morocco \\ abdelwahab.naji@gmail.com
}

\author{
Mohammed Ramdani \\ Department of computer science \\ Faculty of Sciences and Technics of \\ Mohammedia, Hassan II University \\ Morocco \\ ramdani@fstm.ac.ma
}

\begin{abstract}
Recommendation systems are among the most interesting systems which simplify web or search experiences for users by selecting specific items related to their interests from a wide range of choices for them. The use of such systems in e-learning has helped learners filter choices and select content through the utilization of different methods such as the content-based approach and the collaborative approach; however, these methods have not reached an optimal satisfaction among learners about the offer provided. Hence, in this study, we try to propose a different approach based on clustering to make up for the shortcomings of the previously mentioned methods. The main objective of this proposed approach is to get homogeneous groups of learners, and eventually assure that the items recommended are all covered and assimilated by learners.
\end{abstract}

\section{CCS CONCEPTS}

- Theory of computation Unsupervised learning and clustering - Computing methodologies Rule learning • Computing methodologies $\sim$ Vagueness and fuzzy logic

\section{KEYWORDS}

Adaptation, Collaborative, Filtering, Homogeneity, Hybrid, Learning, Personalization, Recommendation System (RS).

\section{ACM Reference format:}

Jamal Mawane, Abdelwahab Naji, Mohammed Ramdani. 2018. Clustering collaborative filtering approach for Diftari E-Learning platform' recommendation system. In SITA'18: 12th International Conference on Intelligent Systems: Theories and Applications, October 24-25, 2018, Rabat, Morocco. ACM, New York, NY, USA, 6 pages

https://doi.org/10.1145/3289402.3289535

\section{INTRODUCTION}

With the advance of web technology as well as the rate of accessibility to internet all over the world, the majority of teachers have pushed the limit of learning outside classrooms or course sessions, so as to align objectives and contents to the different needs of their learners. More importantly, the last few years have seen the emergence of free online courses (MOOC-Massive On

Permission to make digital or hard copies of part or all of this work for personal or classroom use is granted without fee provided that copies are not made or distributed for profit or commercial advantage and that copies bear this notice and the full citation on the first page. Copyrights for components of this work owned by others than ACM must be honored. Abstracting with credit is permitted. To copy otherwise, or republish, to post on servers or to redistribute to lists, requires prior specific permissionand/or a fee. Request permissions from Permissions@acm.org.

SITA'18, October 24-25, 2018, Rabat, Morocco

(C) 2018 Copyright held by the owner/author(s).

ACM ISBN 978-1-4503-6462-1/18/10 ..\$15.00

https://doi.org/10.1145/3289402.3289535
Line Open Courses) sites and other E-Learning platforms, attempting to improve learning offers and widen the range of choices for them. As a result, online learners can now easily find any content they may be looking for, presented in different forms. By developing advanced e-learning environments that are primarily information-based, interactive and constrained by space and time, numerous measures need to be taken to maintain the value of offers and satisfy a large number of learners. In this vein, adaptive education has become an important tool to personalize content in educational systems taking into consideration the large number of students who are the main users of these systems as well as their different backgrounds, goals, abilities and individual traits. Personal learning happens when e-learning systems are designed to fit the needs, goals, talents and interests of learners. It can be achieved by using predefined rules that provide successive learning subjects on the learning path[7,8]. This is also achieved through the use of evidence rules, user models and recommending techniques[1]. Recommender systems are software tools and techniques that provide users with information [2-6]. Suggestions refer to different decision-making processes, such as which items to buy based on selling Statistics, what music to listen to or what content to learn. In this recommendation process, "Item" is a generic term that can be used to indicate what the system recommends to users. Major search engines such as Google and other e-commerce websites, such as Amazon, use recommendation systems in their personal services.

Unfortunately, basic algorithms cannot be directly transferred to the field of e-learning in the same way they can be used with movies or books. One of the major constraints is to take into consideration the cognitive status of the learner and the learning content [13].

This in return raises a pertinent question: Does everything that is proposed deserve to be learned?

In the same way, the winter 2017 statistical results of the MOOCs platforms show that only about $10 \%$ of participants enrolled in a course go to the end; the record held by the Functional Programming in Scala programming course testifies that the results are below expectations: $20 \%$ of the 50,000 participants were certified ${ }^{1}$.

There is obviously no single way to increase the success rate, because each MOOC tries with its own recommendation system to select who you want to touch ${ }^{2}$. The idea developed in this article focuses on this problem and attempts to improve the success rate based on clustering profile collaboration.

\footnotetext{
$1_{\text {http://blog.educpros.fr/matthieu-cisel/2013/06/01/mooc-ce-que-les-taux-dabandon-signifient }}$ 2 http://blog.educpros.fr/matthieu-cisel/2013/05/03/quand-les-profs-simprovisent-webmarketers/
} 
The rest of this paper is organized as follows: In Section 2, we present a related work regarding recommendation system and its usage in E-learning environments. Our approach for recommendation and the recommendation model are explained in Section 3. Section 4 presents the experimental results. Finally, the last Section, will present the concluding remarks and suggestions for future work.

\section{RECOMMENDATION SYSTEM}

In recommendation systems, the collaborative-filtering algorithm and the content-based algorithm use a wide variety of techniques. The classification of these algorithms depends in particular on the type of data used and the learning method used by the recommendation system. In this part, we will try to distinguish between the content-based algorithm and the Collaborative Filtering. At the end of this section, we provide a summaryof both algorithms, as well as a hybrid algorithm form that merges the two algorithms to correct their shortcomings.

\subsection{Content-based recommendation technique}

The content-based recommendation technique is based on the assumption that Objects (Lessons, Exercises, Videos, Movies) with similar content already searched will be similarly requested, and so recommended to the user Fig 1. This technique is based on the analysis of the similarities in content between objects previously viewed by users and those that have not yet been consulted.

Thus, for example, a user who has already looked for loop exercises in a very specific programming language would be interested in other exercises on the same content. The system analyzes the relationships between the different exercises and the one previously seen by this user. This linking is generally evaluated by considering the attributes defining the content itself [9].

Content-based techniques can be classified into two different categories [10-12]

1. Case-based reasoning (CBR) techniques and,

2. Attribute-based techniques

2.1.1 Case-based reasoning (CBR). This is a technique which recommends the objectives that have the highest correlation value with the objectives that the learner covered and validated or liked before. Case-based reasoning is useful for keeping the learner informed about learning objectives. These techniques do not require content analysis, and the quality of the recommendation improves over time as learners interact with more elements.

2.1.2 Attribute-based techniques. This technique is related to relevant Learner profile attributes which could have weight relating to their influence. New attributes or new learners cannot be a problem. The basic technologies are sensitive to the learner profiles [14], where the control and learning personalization are based on their profile attributes, values and weights
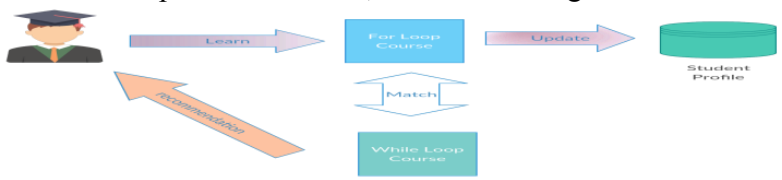

\subsection{Collaborative filtering technique}

The second type of recommendation systems is based on the assumption that users who have liked similar items in the past have similar interests and tastes and will therefore enjoy the same items in the future Fig 2.One of the best examples of such a system has been popularized by Amazon.com, e-commerce websites and its Item-to-item Collaborative Filtering algorithm which translates to the site by the "People Who Bought" feature product $x$ also purchased the product $y^{\prime \prime}$ Table 1 .

The main advantage of this approach is that it does not require precise description of the objects to recommend. Since the recommendations are based on all users' interactions with objects / products. This method makes it possible to recommend complex objects without having to analyze them.

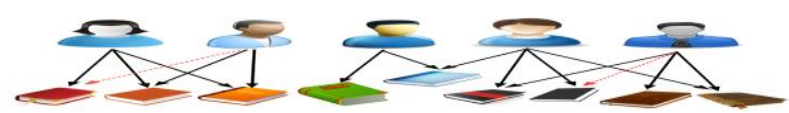

Figure 2: Collaborative filtering technique

Table 1: Learners-Books Rating Matrix

\begin{tabular}{|c|c|c|c|c|c|c|c|c|c|}
\hline $\begin{array}{c}\text { Book } \\
\text { Learners }\end{array}$ & B1 & B2 & B3 & B4 & B5 & B6 & B7 & B8 & B9 \\
\hline L1 & 9 & 10 & 9 & $?$ & $?$ & $?$ & $?$ & $?$ & ? \\
\hline L2 & $?$ & 9 & 10 & $?$ & $?$ & $?$ & $?$ & $?$ & ? \\
\hline L3 & $?$ & $?$ & $?$ & 8 & 9 & $?$ & $?$ & $?$ & $?$ \\
\hline L4 & $?$ & $?$ & $?$ & $?$ & 7 & 8 & 10 & $?$ & 10 \\
\hline L5 & $?$ & ? & $?$ & $?$ & $?$ & 9 & $?$ & 8 & 10 \\
\hline
\end{tabular}

The key step in collaborative filtering methods based on the background of the learner's activities is to define the similarity between Learners or Objectives Table 1. In this case, several methods can be used to calculate the similarities, the most used are: Pearson correlation [15] and cosine similarity [16].The Pearson correlation between two learners' $L u$ and $L v$ is calculated as follows:

$\mathrm{S}_{1}(L u, L v)=\frac{\sum_{\mathrm{j}}^{\mathrm{n}}\left(\mathrm{r}_{\mathrm{Lu}, \mathrm{j}}-\overline{\mathrm{r}}_{\mathrm{Lu}}\right)\left(\mathrm{r}_{\mathrm{Lv}, \mathrm{j}}-\overline{\mathrm{r}}_{\mathrm{Lv}}\right)}{\sqrt{\sum_{\mathrm{j}}^{\mathrm{n}}\left(\mathrm{r}_{\mathrm{Lu}, \mathrm{j}}-\overline{\mathrm{r}}_{\mathrm{Lu}}\right)^{2} \sum_{\mathrm{j}}^{\mathrm{n}}\left(\mathrm{r}_{\mathrm{Lv}, \mathrm{j}}-\overline{\mathrm{r}}_{\mathrm{LV}}\right)^{2}}}$

Also the similarity between two learners' $L u$ and $L v$ with Cosine similarity method can be calculated as follows:

$\mathrm{S}_{2}(L u, L v)=\frac{\sum_{\mathrm{j}}^{\mathrm{n}} \mathrm{r}_{\mathrm{Lu}, \mathrm{j}} * \mathrm{r}_{\mathrm{Lv}, \mathrm{j}}}{\sqrt{\sum_{\mathrm{j}}^{\mathrm{n}} \mathrm{r}_{\mathrm{Lu}, \mathrm{j}}^{2}} \sqrt{\sum_{\mathrm{j}}^{\mathrm{n}} \mathrm{r}_{\mathrm{Lv}, \mathrm{j}}^{2}}}$

In two case: $\mathrm{r}_{\mathrm{Lu}, \mathrm{j}}$ is the rating of the $\mathrm{jth}$ Book by Learner $\mathrm{Lu}, \overline{\mathrm{r}}_{\mathrm{Lu}}$ is the average rating of Learner $\mathrm{Lu}$, and $\mathrm{S}(L u, L v)$ is The similarity between user $L u$ and user $L v$ using one of two methods.

For example, the Person and cosine similarity computation for the case shown in Fig 2associated to Learners-Books Rating Matrix Table labove is presented in the table below Table 2

Figure 1: Content-based recommendation technique 
Clustering collaborative filtering approach for Diftari E-Learning platform' recommendation system.

Table 2: Pearson - Cosine Correlation Computation

\begin{tabular}{|l|ccccc|ccccc|}
\multirow{2}{*}{ Learners } & \multicolumn{4}{|c|}{ Pearson Correlation } & \multicolumn{5}{|c|}{ Cosine Correlation } \\
\cline { 2 - 11 } \multicolumn{1}{|c|}{} & L1 & L2 & L3 & L4 & L5 & L1 & L2 & L3 & L4 & L5 \\
\hline L1 & 1,00 & 0,77 & $-0,38$ & $-0,62$ & $-0,50$ & 1,00 & 0,83 & 0,00 & 0,00 & 0,00 \\
L2 & 0,77 & 1,00 & $-0,28$ & $-0,47$ & $-0,37$ & 0,83 & 1,00 & 0,00 & 0,00 & 0,00 \\
L3 & $-0,38$ & $-0,28$ & 1,00 & $-0,02$ & $-0,37$ & 0,00 & 0,00 & 1,00 & 0,30 & 0,00 \\
L4 & $-0,62$ & $-0,47$ & $-0,02$ & 1,00 & 0,28 & 0,00 & 0,00 & 0,30 & 1,00 & 0,55 \\
L5 & $-0,50$ & $-0,37$ & $-0,37$ & 0,28 & 1,00 & 0,00 & 0,00 & 0,00 & 0,55 & 1,00 \\
\hline
\end{tabular}

As shown in Table 2 above, the two learners L1 and L2 Fig 2 have a strong similarity, proven by both methods, which explains the proposition of 'book1' to 'learner L2', the same case occurs between L4 and L5, with a medium correlation leading to the recommendation of Book5 for learner L5.

\subsection{Comparing recommendation techniques}

Both quoted techniques represent a big field for scientific research in the aim of improving them through reducing the shortcomings of every technique and prospecting new uses.

Table 3: The following table raises an overview of these two techniques

\begin{tabular}{|c|c|c|c|}
\hline Technique & Variant & Advantages & Problems \\
\hline 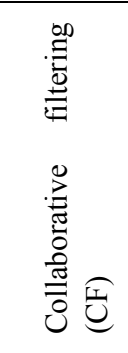 & $\begin{array}{l}\text { User } \\
\text { Collaboration } \\
\text { Item } \\
\text { Collaboration } \\
\text { User/Item } \\
\text { Collaboration }\end{array}$ & $\begin{array}{l}\text {-Domain } \\
\text { knowledge } \\
\text { not needed } \\
\text {-Adaptive: } \\
\text { quality } \\
\text { improves } \\
\text { over time } \\
\text {-Implicit } \\
\text { feedback } \\
\text { sufficient }\end{array}$ & $\begin{array}{l}\text { Scale } \\
\text { Sparse } \\
\text { data } \\
\text { Cold-Start } \\
\text { Popularity } \\
\text { bias }\end{array}$ \\
\hline 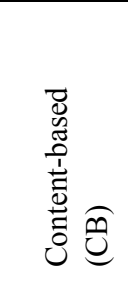 & $\begin{array}{l}\text { Case based } \\
\text { reasoning } \\
(\mathrm{CBR}) \\
\text { Attribute- } \\
\text { based } \\
\text { techniques }\end{array}$ & $\begin{array}{l}\text { Sensitive to } \\
\text { changes of } \\
\text { preference } \\
\text { Can } \\
\text { recommend } \\
\text { new product }\end{array}$ & $\begin{array}{l}\text { New user } \\
\text { ramp-up } \\
\text { problem } \\
\text { Quality } \\
\text { dependent } \\
\text { on large } \\
\text { historical } \\
\text { data set. }\end{array}$ \\
\hline
\end{tabular}

As the table above-Table 3-shows, both techniques do not have the same shortcomings, which suggested to the researchers to propose a hybrid system.

\subsection{Hybrid recommender technique}

Hybrid recommender systems associate more than one recommendation technique to enhance performance with fewer of the drawbacks associated to each technique. Recent research using the hybrid recommender systems, combining collaborative filtering and content-based filtering, has given better results in most cases. Hybrid approaches can be implemented in many ways: by making content-based and collaborative-based predictions separately and then combining them, by adding
SITA'18, October 24-25, 2018, Rabat, Morocco

content-based capabilities to a collaborative-based approach, or by unifying the approaches Fig 3into one model[17,18].

Monolithic strategy

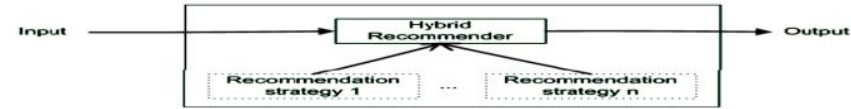

Parallel strategy

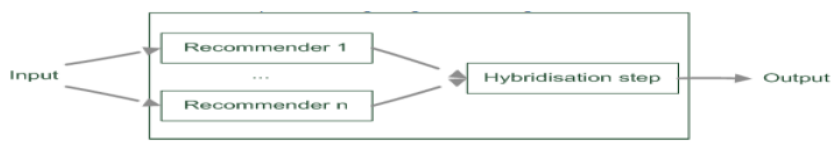

Pipeline strategy

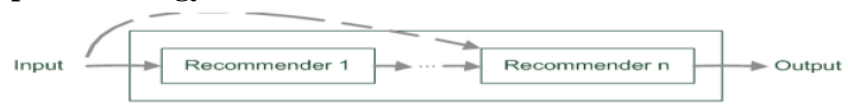

Figure 3:Hybrid recommender technique strategies

\section{COLLABORATIVE CLUSTRING RECOMMENDATION}

As it was mentioned in the introduction, a recommendation system may offer various types of content to learners. Nevertheless, not all that is offered can be useful. This paper, outlines anew collaborative filtering algorithm approach Fig 4, developed for Diftari e-learning platform ${ }^{3}$, this recommendation approach based on the clustering method to pre-produce learners' models, and Scoring and Sorting step to find the most interesting content to recommend. The clustering step focused on three aspects of learners, their personal information, their previous achievement outcomes, and their interaction with the system. The items to recommend will be chosen among the most scored items in the same cluster of homogeneous students, in order to provide better recommendation than traditional collaborative filtering. In the rest of this section, we present this approach step by step.

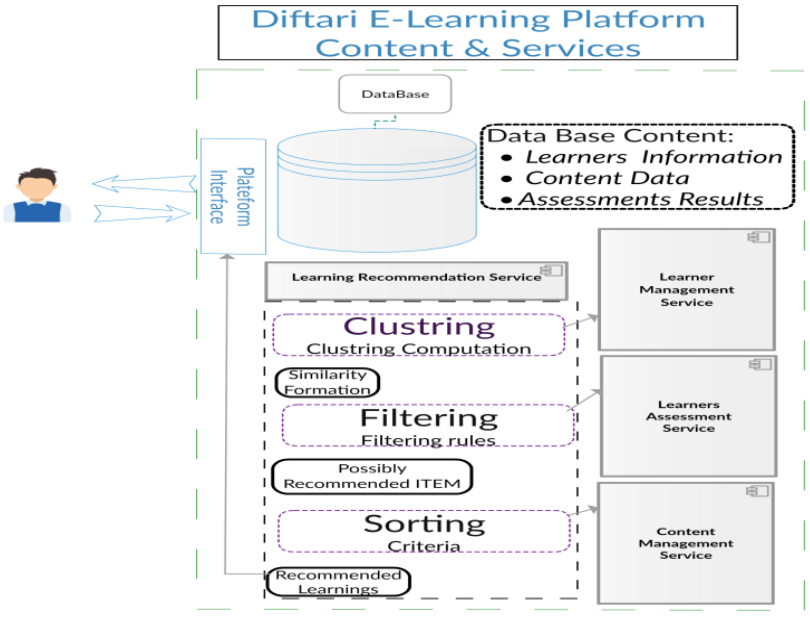

Figure 4: System Architecture.

\footnotetext{
${ }^{3}$ Diftari E-learning Platform: http://www.amsi.ma/diftari/
} 


\subsection{Pre-processing and Merging Data}

Data about learners were collected explicitly and implicitly from their activities in E-learning sessions. The first step consists of cleaning, to pre-process and merge the information about every learner. In fact, the cleaning, pre-processing and merging from different data bases are the most important tasks to prevent data anomalies such as missing, redundant or noisy data.

\subsection{Clustering and Filtering}

The clustering problem is a well- researched issue in the computer field due to its incredible variety of applications, KMeans, KMedians and Fuzzy KMeans are widely popular methods to clustering data. For all, the objective is to find the homogeneous $\mathrm{K}$ group of similar data point to each other for which the sum of the distance between a center and all points in the cluster is minimized. In this part, which is the first step of our approach, the objective is to find the optimal value of $\mathrm{K}$ and then perform the clustering method to get homogeneous groups of learners similar to each other.

3.2.1 Finding Optimal $\mathrm{K}$ with Elbow Method. One of the largest used metrics to find an optimal value of $\mathrm{K}$ is the mean distance between data points and their cluster centroid. Since increasing the number of clusters will always reduce the distance to data points, increasing $\mathrm{K}$ will always decrease this metric to the extreme of reaching zero when $\mathrm{K}$ is the same as the number of data pointsFig 5.The main idea of this method is to relate the mean distance to the centroid to a function of $\mathrm{K}$.

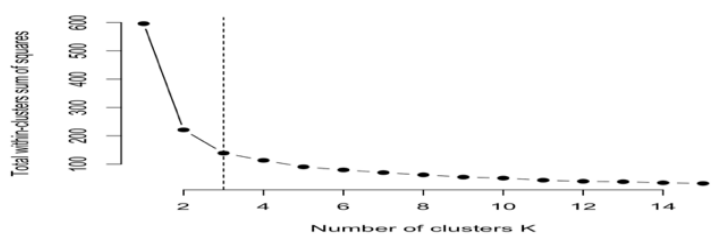

Figure 5: The Elbow graph to determine the optimal $k$

\subsubsection{Clustering Methods}

3.2.2.1 KMeans Algorithm. KMeans clustering is one of the simplest types of unsupervised learning, which is used with numeric data. The main objective of this algorithm is to find $\mathrm{K}$ groups based on the data points distances and on features similarity. The algorithm works iteratively to assign each data point to one of $\mathrm{K}$ groups based on the features that are provided

3.2.2.2 KMedians Algorithm. With a given data set, the $\mathrm{k}$-medians algorithm attemptsto create $\mathrm{k}$ disjoint cluster that minimizes the total 1-norm distance from each point to its nearest cluster center. KMedians is quite similar to KMeans.

3.2.2.3Fuzzy KMeans Algorithm[19]. The fuzzy KMeans(FKM) algorithm is a clustering algorithm developed by Dunn 1973, and later on improved by Bezdek in 1981. It needs predetermined number of clusters; thus, the aim of the algorithm is to put each data point to one of the clusters, but the specificity of FKM is that it does not relate the data point only to a given cluster; instead, it calculates the degree of membership that a data point will belong to that cluster. FKM can be extremely fast because the number of iterations required to achieve a specific clustering exercise corresponds to the required accuracy.
3.2.3 Scoring and Sorting.

In this step, the objective is to detect the learning objectives that were not covered by the user, and then to filter in this list the most successful learning according to two criteria: the degree and the number of successes.

3.2.4 Process

Step $\mathrm{N}^{\circ} 1$ : Using clustering method to find students who had the same profile.

Step $\mathrm{N}^{\circ}$ 2: Find all courses well succeeded by the others.

Step $\mathrm{N}^{\circ}$ 3: Scoring the list of founded courses.

Step $\mathrm{N}^{\circ} 4$ : Sorting the list.

Step $\mathrm{N}^{\circ}$ : Present to current student then $\mathrm{N}$ most founded list. 3.2.5 Data Set

In order to test the validity of our approach, and because our elearning platform does not currently contain a large amount of data, for our study we use a real-world case data set, certified by the Open Data Institute, named the anonymized Open University Learning Analytics Dataset (OULAD), which contains information about 22 courses, 32,593 students, their assessment results, and logs of their interactions with the Virtual Learning Environment (VLE) for seven selected courses (called modules) represented by daily summaries of student clicks $(10,655,280$ entries). The dataset consists of tables connected using unique identifiers. All tables are stored in the csv format ${ }^{4}$.

\section{EXPERIMENTATION}

This experiment is conducted to test the usefulness of our approach by determining which clustering method is more powerful for our data Set and which interesting recommendation Items are selected for every learner.

All results presented below are concluded on an Intel Core i5 1.4 GHz, with 4 GB DDR3 memory, and using Python 3.6.3 as the scripting language

\subsection{Input description:}

In order to get the best clustering model, all students' features were cleaned and merged from two csv files of Data Set so as to cluster different features of the three personality aspects (personal information, previous achievement outcomes, and interaction with the system) for processing. The features selected for experience are shown in the figureFig 6below:

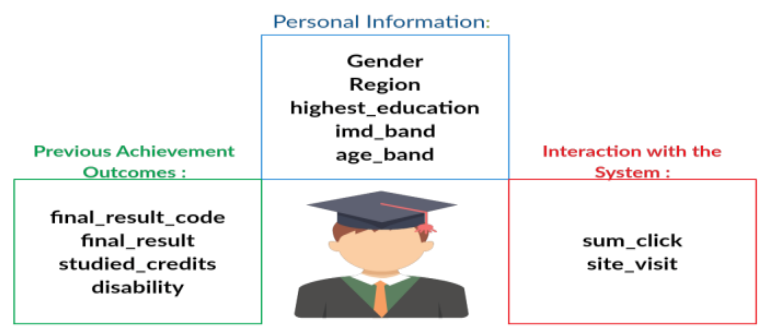

Figure 6: The three learners' information aspects.

${ }^{4}$ Kuzilek J., Hlosta M., Zdrahal Z. Open University Learning Analytics dataset Sci. Data 4:170171 doi: 10.1038/sdata.2017.171 (2017). 
Clustering collaborative filtering approach for Diftari E-Learning platform' recommendation system.

\subsection{Clustering:}

In this step, we focus our experimentation on two pressing questions related to clustering, which are:

Which Value of $\mathrm{k}$ to use?

Which methods to choose?

4.2.1 Number of Clusters. The Elbow method defined in paragraph [3.2.1] is applied to the used Data Set and the result is given in figure belowFig 7 .

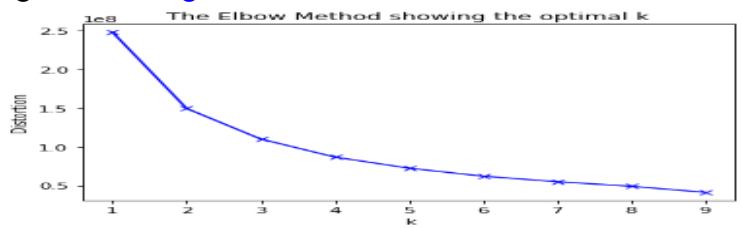

Figure 7: The Elbow Method results below to the Data Set.

Like shown inFig 7the optimal clusters number for our data set below with the elbow method is $\mathrm{K}=4$, which main that we have only four homogenous learner groups.

4.2.2 Choosing a Clustering Method. To make the good choice, a study comparative on the three methods of clustering based on three axes: firstly, their results are crossed to estimate the compatibility Table 4,then the estimated average time of execution is presented in Table 5in the aim of detecting the fastest method.

4.2.3Clusters results. The results displayed in Table 4 give us an idea of the compatibility of the results of each.

Table4: Clustering Distribution.

\begin{tabular}{|c|c|c|c|c|c|c|c|c|c|}
\hline & \multirow[b]{2}{*}{$\begin{array}{l}\text { Clus } \\
\text {-ter }\end{array}$} & \multicolumn{4}{|c|}{ KMedians } & \multicolumn{4}{|c|}{ Fuzzy KMeans } \\
\hline & & 1 & 2 & 3 & 4 & 1 & 2 & 3 & 4 \\
\hline \multirow{4}{*}{$\sum_{1}^{\infty}$} & 1 & $53 \%$ & $47 \%$ & & & $9 \%$ & $91 \%$ & & \\
\hline & 2 & $36 \%$ & & & $64 \%$ & $91 \%$ & & & $9 \%$ \\
\hline & 3 & & $29 \%$ & $71 \%$ & & & $7 \%$ & $93 \%$ & \\
\hline & 4 & & & & $100 \%$ & & & & $100 \%$ \\
\hline
\end{tabular}

The results obtained from our case allow us to deduce that the algorithms of KMeans and Fuzzy KMeans compared to that of KMedian search similar results, with a strong correlation between KMeans and Fuzzy KMeans, for a time of significantly shorter calculation.

Table 5: Execution Time

\begin{tabular}{|l|l|l|l|}
\hline Algorithm & kMeans & KMedians & Fuzzy KMeans \\
\hline Time $(\mathbf{s}) \approx$ & 1.20 & 0.5 & 0.5 \\
\hline
\end{tabular}

Fuzzy KMeans' algorithm is the fastest. In addition to bringing its results closer to the other algorithms, it has a very considerable advantage in the evolution of the system, since cluster
SITA'18, October 24-25, 2018, Rabat, Morocco

membership is not binary, which will favor the discovery of new items for clusters defined at the outset.

Nevertheless, the KMeans algorithm, despite being the heaviest algorithm for our study, can be used as a data discovery algorithm and it can find the optimal $\mathrm{k}$ value.

\subsection{Scoring and Sorting:}

To test the third and the fourth steps of our approach, we take a learner randomly, and we visualize part of his history and what the system recommends, using Fuzzy KMeans Clusters.

Table 6 shows the value of learner features, Table 7 shows a part of learner assessment history, and at last Table 8 shows recommended Items focusing Fuzzy clustering method.

Table 6: Randomly chosen learner information:

\begin{tabular}{|c|c|}
\hline \multicolumn{2}{|l|}{ Feature: Value } \\
\hline Gender: & studied_credits: 240 \\
\hline Region: & disability_Code: $\mathbf{0}$ \\
\hline highest_education: $\mathbf{3}$ & final_result_code: 2 \\
\hline imd_band: 2 & final_result: Distinction \\
\hline age_band: 1 & ClassKmeans: \\
\hline mean_click: 2.73 & ClassFuzzy: \\
\hline 163690644.0 & ClassKmedians: \\
\hline
\end{tabular}

Table 7: History of learning Items

\begin{tabular}{|c|c|c|c|c|}
\hline こ葛 & 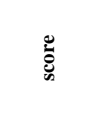 & ঠீ & 胥 & 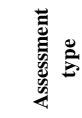 \\
\hline 15015 & 100.0 & BBB & 2014B & CMA \\
\hline 15017 & 100.0 & BBB & 2014B & CMA \\
\hline 15016 & 80.0 & BBB & 2014B & CMA \\
\hline 15018 & 80.0 & BBB & 2014B & CMA \\
\hline 15019 & 80.0 & BBB & 2014B & CMA \\
\hline
\end{tabular}

Table 8: Recommended learning Items

\begin{tabular}{|c|c|c|c|c|c|}
\hline こ & 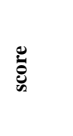 & 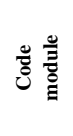 & 范 & 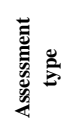 & 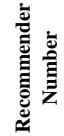 \\
\hline 15020 & 100.0 & BBB & 2014J & TMA & 11 \\
\hline 14991 & 100.0 & $\mathrm{BBB}$ & 2013B & CMA & 5 \\
\hline 14992 & 100.0 & $\mathrm{BBB}$ & 2013B & CMA & 3 \\
\hline 34905 & 100.0 & FFF & $2014 \mathrm{~J}$ & CMA & 1 \\
\hline
\end{tabular}

\subsection{Classical Collaborative Filtering Recommendation:}

In order to confirm our approach, we applied the collaborative recommendation algorithm based on the cosine similarity method on the same Data Set. Table 9 displays the recommendation algorithm result. 
Table 9: Recommended learning Items

\begin{tabular}{|c|c|c|c|c|c|}
\hline こ & 总 & 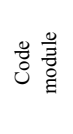 & 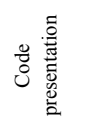 & 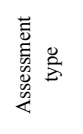 & 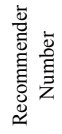 \\
\hline 15015 & 100.0 & $\mathrm{BBB}$ & 2014B & CMA & 1 \\
\hline 14991 & 100.0 & BBB & 2013B & CMA & 1 \\
\hline 15011 & 95.0 & BBB & 2014B & TMA & 1 \\
\hline 14985 & 80.0 & BBB & 2013B & TMA & 1 \\
\hline 14996 & 76.0 & BBB & 2013J & TMA & 1 \\
\hline
\end{tabular}

The Table 9 above shows that the proposed list has items with a score less than 100, and each recommended element by only one learner, which does not make sure a high success rate for learners. The goal of our approach is to improve the success rate of the recommended elements, based on the learners' profile, their previous results, and the most successful elements.

\section{CONCLUSIONS}

In E-learning context, recommender systems may be one of the most important technologies used to improve individual and personalized learning. In this paper, we propose an approach of recommendation for E-learning environments based on collaborative filtering and clustering methods to improve the classical recommendation system which is based on ranking similarity in order to get an efficient learning experience, for both, learners having problem and difficulty to progress in their learning path, and those with normal progress. Firstly, the clustering is based on three characters of students, personal information, results, and interactivity with the system.

Secondly, we took learning items from the same learning clusters, which were not yet covered. And finally, the list of the most appropriate learning item was obtained using the two sorting criteria.

The great concern of researchers when it comes to the subject of recommendation in e-Learning is the non-existence of real open active platforms to test the validity of the proposed metrics and approaches, but the availability of open databases in E-learning simplified the tasks in order to evaluate the accuracy of the recommendation of the proposed models. In our study we used these extracted databases in an online learning environment. The results show that the use of the proposed approach simplified the recommendation and increased the certainty rate of learning success elements because the collaborative Items come from another learner who is very close to the learner used based on the three aspects clustering already mentioned above.

Moreover, our approach can be used to avoid collaborative recommendation technique' problem known as 'cold Start', because any learner can be matched to a cluster which makes it possible to recommend other Items for him.

In the future, our first objective will be to test the implementation of our method in Diftari e-learning platform in real context evolving in time, allowing us later to discover learners' profiles latent parameters (learning style, Emotional intelligence,...). As a second objectives, we will work on improving the sorting and selection criteria (step $2 \& 3$ ) of our approach.

\section{ACKNOWLEDGMENTS}

Knowledge Media Institute, The Open University, Milton Keynes, MK7 6AA, United Kingdom.

\section{REFERENCES}

[1] Paul Resnick and Hal R. Varian. 1997. Recommender systems. Commun. ACM 40, 3 (March 1997), 5658.DOI=http://dx.doi.org/10.1145/245108.245121

[2] Francesco Ricci, LiorRokach, BrachaShapira, and Paul B. Kantor. 2010. Recommender Systems Handbook (1st ed.). Springer-Verlag, Berlin, Heidelberg.DOI=http://dx.doi.org/10.1007/978-1-4899-7637-6

[3] Aleksandra Klašnja-Milićević, BobanVesin, Mirjana Ivanović, Zoran Budimac, Lakhmi C Jain. 2017. Recommender Systems in E-Learning Systems Environments. Springer International Publishing, Cham, 2017,51- 75.

[4] Mobasher, B.::Brusilovsky, P., Kobsa A., Nejdl, W. (eds.) Data mining for web personalization In The Adaptive Web: Methods and Strategies of Web.

[5] J. Bobadilla, F. Ortega, A. Hernando, and A. Gutierrez. Recommender systems survey. Knowledge-Based Systems, 46(0):109 - 132, 2013

[6] J. Lu, D. Wu, M. Mao, W. Wang, and G. Zhang. Recommender system application developments: a survey. Decision Support Systems, 74:12-32, 2015

[7] Hendrik Drachsler, Hans G. K. Hummel, and Rob Koper. 2008. Personal recommender systems for learners in lifelong learning networks: the requirements, techniques and model. Int. J. Learn. Technol. 3, 4 (July 2008), 404-423. DOI=http://dx.doi.org/10.1504/IJLT.2008.019376

[8] A. Naji,M. Ramdani. 2013.Using the Ant Colony Algorithm to Establish the Best Path of Learning Activities. Applied Mathematical Sciences, Vol. 7, 2013, no. 78, 3873 - 3881 HIKARI Ltd, http://dx.doi.org/10.12988/ams.2013.35282

[9] Gediminas Adomavicius and Alexander Tuzhilin. 2005. Toward the Next Generation of Recommender Systems: A Survey of the State-of-the-Art and Possible Extensions. IEEE Trans. on Knowl. and Data Eng. 17, 6 (June 2005), 734-749. DOI: https://doi.org/10.1109/TKDE.2005.99

[10] Schmitt S, Bergmann R. 1999. Applying case-based reasoning technology for product selection and customization in electronic commerce environments. In: 12 th bled electronic commerce conference, vol. 273

[11] Yong Zheng,BamshadMobasher, Robin Burke. 2015. Similarity-Based Context-Aware Recommendation. Proceedings, Part I, of the 16th International Conference on Web Information Systems Engineering --- WISE 2015, p.431447, November 01-03, 2015. DOI:10.1007/978-3-319-26190-4_29

[12] Stefano Aguzzoli, Paolo Avesani, and Paolo Massa. 2002. Collaborative CaseBased Recommender Systems. In Proceedings of the 6th European Conference on Advances in Case-Based Reasoning (ECCBR '02), Susan Craw and Alun D. Preece (Eds.). Springer-Verlag, London, UK, UK, 460-474.

[13] Edward Rolando Núñez-Valdéz, Juan Manuel Cueva Lovelle, Oscar SanjuánMartínez, Vicente García-Díaz, Patricia Ordoñez de Pablos, and Carlos Enrique Montenegro Marín. 2012. Implicit feedback techniques on recommender systems applied to electronic books. Comput. Hum. Behav. 28, 4 (July 2012), 1186-1193. DOI=http://dx.doi.org/10.1016/j.chb.2012.02.001

[14] Drachsler, H., Hummel, H. G. K., \& Koper, R. 2008. Personal recommender systems for learners in lifelong learning: requirements, techniques and model International Journal of Learning Technology 3(4), $404-423$.

[15] G. Sidorov, A. Gelbukh, H. Gómez-Adorno, and D. Pinto. 2014. Soft Similarity and Soft Cosine Measure: Similarity of Features in Vector Space Model. Computación y Sistemas. 2014, vol.18, n.3, 491---504. http://dx.doi.org/10.13053/CyS-18-3-2043

[16] J. Hauke, T. Kossowski. 2011. Comparison of values of Pearson's and Spearman's correlation coefficient on the same sets of data. QuaestionesGeographicae 30(2), BoguckiWydawnictwoNaukowe, Poznań 2011, pp. 87-93. DOI 10.2478/v10117-011-0021-1.

[17] Francesco Ricci, LiorRokach, BrachaShapira. 2015. Introduction to Recommender Systems Handbook. Recommender Systems Handbook (2nd ed.), pp. 1-35. Springer. 2011. DOI 10.1007/978-1-4899-7637-6

[18] Robin Burke. 2002. Hybrid Recommender Systems: Survey and Experiments. User Modeling and User-Adapted Interaction 12, 4 (November 2002), 331370. DOI=http://dx.doi.org/10.1023/A:1021240730564

[19] R. Yera and L. Martínez, "Fuzzy Tools in Recommender Systems: A Survey ", International Journal of Computational Intelligence Systems, vol. 10, issue 1, pp. $776-803,2017$. 